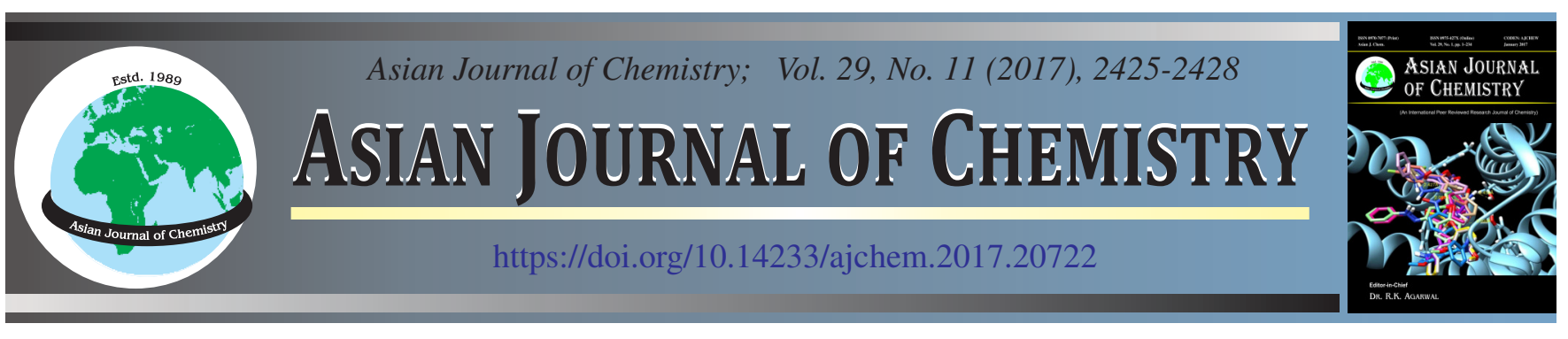

\title{
Extraction and Characterization of Preformed Mixed Phase Graphene Sheets from Graphitized Sub-Bituminous Coal
}

\author{
Akshatha A. Rao, Ashlin M. Raj and B. Manoj
}

Department of Physics, Christ University, Bengaluru-560 029, India

*Corresponding author: E-mail: manoj.b@christuniversity.in

\begin{abstract}
In present paper, a facile method is reported to extract mixed phase nanometre-sized carbon sheets from sub-bituminous coal. The lattice constants $\left(\mathrm{L}_{\mathrm{a}}\right.$ and $\mathrm{L}_{\mathrm{c}}$ ) of sub-bituminous coal were calculated to be 4.82 and $1.41 \mathrm{~nm}$, respectively. The aromatic layers and average number of carbon atoms in the aromatic lamellae were estimated as 5 and 8, respectively. The obtained graphene sheets exhibits broadened $\mathrm{D}$ and $\mathrm{G}$ band in addition to a very broad 2D bump. Defect to graphitic ratio is found to be 0.54 indicating less disorder in graphene nanomaterial formed. This is further corroborated by $\left(\mathrm{I}_{\mathrm{D}} / \mathrm{I}_{\mathrm{D}^{\prime}}\right)$ ratio which was observed to be 3.40 , confirming the defect has originated from boundary. The SEM analysis reveals the formation of large number of carbon layers with different shape in the nanometer scale range. Formation of graphene dots in the shape of hexagonal, spherical, graphene layers and corn shaped carbon nanotubes are noticed in the TEM image.
\end{abstract}

Keywords: Nano-carbon, Defected carbon, Graphene dots, Coal.

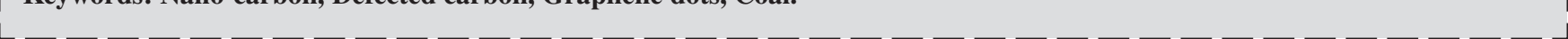

\section{INTRODUCTION}

Graphene-wonder material of carbon is a monolayer, hexagonal structure having exceptional physical, chemical and mechanical properties [1-3]. Graphene is synthesized from variety of precursors like graphite, graphene oxide, carbon nanotubes, carbon fibres and activated carbon. Physical approach such as mechanical exfoliation, lithography and other approaches such as hydrothermal treatment, electrochemical oxidation, proton ablation and chemical oxidation have been used for the synthesis of graphene [4,5]. However, most of the above stated precursors and production methods are too expensive and are optimized for the production of graphene with a homogenous $s p^{2}$ phase [6,7].

For applications, defect is introduced in graphene which enhances its property and this could be extrinsic, intrinsic or mixed phase of $s p^{2}-s p^{3}$ carbon network. The presence of amorphous carbon content along with graphene shows the properties of mixed structure. It is reported that mixing of graphene with amorphous carbon enhances the hardness and elastic property significantly. Defect in graphene system is anything that its symmetry of honeycomb lattice. This can be attributed to edge effect, grain boundary, vacancy or change in hybridization. This defect has a major role on the properties of graphene $[8,9]$. The research on the abundant source of carbon-coal-reveals that it contains graphite-like clusters and poly-aromatic structures similar to $s p^{2}$ bonding properties of graphene $[6,7,10,11]$. It also reveals that carbon in coal has turbostratic structure that of graphite and amorphous carbon and this could be separated by simple method like chemical oxidation $[7,11]$. These nanocrystallites of coal are confined to a small area and hence very tedious to extract. Hence, an effective process of demineralization is necessary to understand turbostratic structure of coal. For this purpose, hydrofluoric acid (HF) was found to be the best chemical than other reagents since it removes almost all minerals in coal [12]. Herein, we present the effective synthesis of mixed phase graphene from the low grade fossil fuel-coal by the method of chemical oxidation using hydrofluoric acid.

\section{EXPERIMENTAL}

A lump of Indian sub-bituminous coal was powdered and from which $5 \mathrm{~g}$ of sample and was pre-treated with chelating agent (EDTA) for $24 \mathrm{~h}$. The treated coal was filtered and the residue was dried in vacuum oven. About $2 \mathrm{~g}$ of the sample was mixed with aqueous HF (40\%) in a polypropylene beaker for $2 \mathrm{~h}$. The mixture was further ultrasonicated for $30 \mathrm{~min}$ and left for gravity separation. The mixture was washed with deionized water several times to remove acid content. It was further centrifuged at $5000 \mathrm{rpm}$ to separate the residue and the supernatant. The residue was dried in the vacuum oven at $110{ }^{\circ} \mathrm{C}$ to obtain a dry powder of leached coal to investigate 
the graphene nanostructures. The chemicals used were analytically pure and used as received.

Characterization methods: X-ray powder diffraction (XRD) profile were obtained from a Bruker AXS D8 Advance $\mathrm{X}$-ray spectrometer using $\mathrm{CuK}_{\alpha}$ radiation. Raman spectra were measured using Lab RAM HR spectrometer while the Fourier transform infrared (FTIR) spectra were recorded from a Thermo Nicolet 370 spectrophotometer. The atomic structure of GOQDs was investigated by high resolution transmission electron microscopy (TEM) using JEOL/JEM 2100 system. SEM micrographs and EDS spectra were recorded with GEMINI ULTRA 55 high resolution microscope.

\section{RESULTS AND DISCUSSION}

The XRD profile of graphitized coal two peaks namely $\gamma$-band and $\pi$-band (at $2 \theta=18.29^{\circ}$ and $24.85^{\circ}$ as in Fig. 1). The $\pi$-band originate from aromatic ring while $\gamma$-band from aliphatic chains. The symmetric (002) band around $24^{\circ}$ indicated the existence of $\pi$-band and broad band at $18.29^{\circ}$, confirming the saturated structures at the edge of crystallites. The higher intensity in the low angle range is attributed to inter-particle and small angle scattering by fissures, nanopores, heterogeneities, etc. present in the carbon lattice $[8,11]$. The diffraction centered around $26^{\circ}$ is mainly indicating the presence of amorphous content along with crystalline carbon.

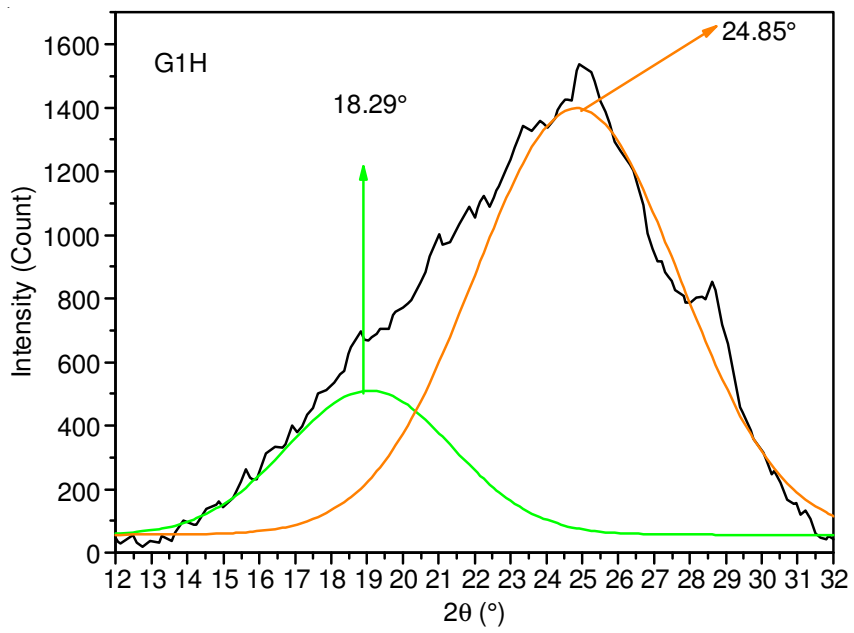

Fig. 1. X-ray analysis of graphene layers from sub-bituminous coaldeconvoluted spectrum

Lateral size $\left(\mathrm{L}_{\mathrm{a}}\right)$ and stacking height $\left(\mathrm{L}_{\mathrm{c}}\right)$ were calculated and are found to be $4.82 \mathrm{~nm}$ and $1.41 \mathrm{~nm}$, respectively. The average number of aromatic layers $(\mathrm{N})$ and average number of carbon atoms per aromatic lamella (n) are estimated as 5 and 8 , respectively. The d-spacing $\left(\mathrm{d}_{002}\right)$ is found to be 0.353 $\mathrm{nm}$, which is in concurrence with the previous reported value for graphene like system [11].

Raman analysis (Fig. 2) shows features at 1148, 1274 and $1307 \mathrm{~cm}^{-1}$ (ta-C) attributed $s p^{3}$ carbon. Previous studies $[3,13]$ assigned this band to hexagonal diamond, nanocrystalline diamond or $s p^{3}$ rich carbon structures. Bacsa et al. [14] labelled the origin of this band to mixed structure of $s p^{2}-s p^{3}$ network. We assign this band to $s p^{2}-s p^{3}$ mixed structure which is supporting the XRD analysis result.

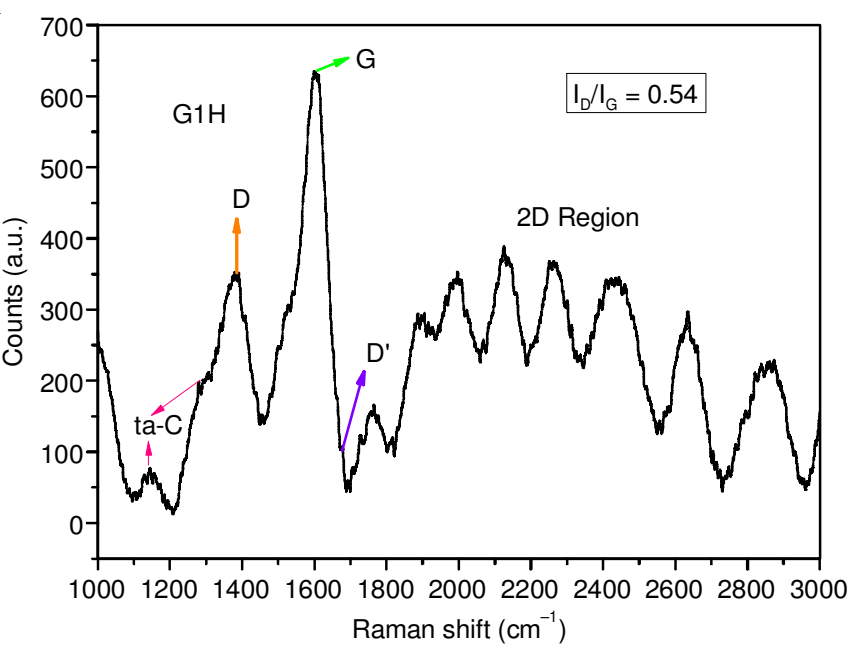

Fig. 2. Raman spectra of leached sample

The in-plane vibrational mode of $s p^{2}$ hybridized carbon atoms of graphene nanostructures are observed at $1582 \mathrm{~cm}^{-1}$. Empirically, the band position can be correlated to the number of aromatic layers present in the sample:

$$
\omega_{\mathrm{G}}=1581.6+11 /\left(1+\mathrm{n}^{1.6}\right)
$$

where ' $\omega_{\mathrm{G}}$ ' is position of band in wavenumber and ' $n$ ' is number of layers present in the sample [15-17]. Number of layers is enumerated with the eqn. 1 and is found to be 5 which is agreeing with the results of XRD analysis.

Other two noticeable peak, D and D', are attributed to the defect-induced Raman features, which is absent in highly crystalline graphite. The D band (at $1380 \mathrm{~cm}^{-1}$ ) is due to the delocalized $\pi$ states around the $s p^{2}$ chains $[4,15,18]$. The intensity ratio $\left(\mathrm{I}_{\mathrm{D}} / \mathrm{I}_{\mathrm{G}}\right)$ is a measure of defect quantity in graphitic materials is found to be 0.54 indicating low defect. The intensity ratio $\left(\mathrm{I}_{\mathrm{D}} / \mathrm{I}_{\mathrm{D}}\right)$ gives the information about nature and concentration of defects. This was found to be 3.40 and refers to boundary-like defects as reported by Eckman et al. [16] in graphene system.

The large number of defects and disorder can lead to defect activated bands such as D band and an overtone of D-2D band. These bands may lead to a broad peak due to merging and result as a bump like shape for the 2D band [19,20]. The 2D band is fitted with multiple Lorentian profile has 6 peaks, the 3 intense peaks are $\mathrm{G}^{*}, \mathrm{G}^{\prime}, \mathrm{D}+\mathrm{D}^{\prime}$ bands at 2431, 2630 and $2859 \mathrm{~cm}^{-1}$ and other peaks are cosidered to be residual peaks (Fig. 3) [1,4,9,17,21].

The position of G' band is a function of number of layers and order of stacking of the graphite sample. For a perfectly stacked few layer graphene (about 6-8 layers), the 2D peak (G' peak) exhibits two peak profile [21]. With increase of disorder, the G' shoulder shift upwards and finally erges with G' band resulting a single $2 \mathrm{D}$ band ( $\mathrm{G}^{\prime}$ band). Thus, the Raman analysis showed that after chemical oxidation the structure of carbon in sub-bituminous coal was converted into a mixed phase graphene nanostructure containing both $s p^{2}$ and $s p^{3}$ components. The Raman analysis also confirms the presence of multilayer graphene nanosheets that contain mixed phase $s p^{2}-s p^{3}$ components than a pure $s p^{2}$ graphene layer. 


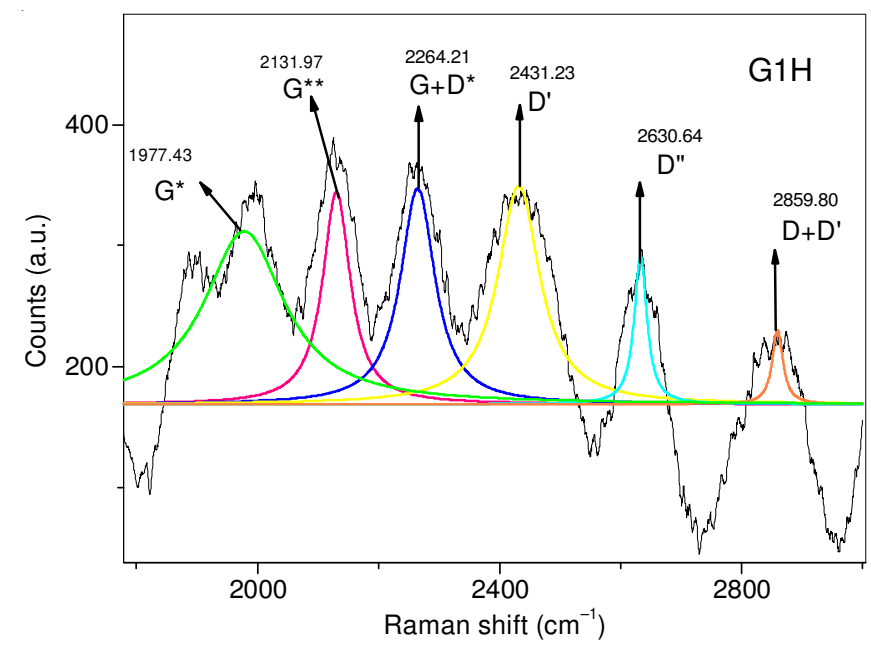

Fig. 3. Second order Raman spectrum

FTIR spectra of the sample $(\mathrm{G} 1 \mathrm{H})$ are as shown in the Fig. 4. The band at $3443.41 \mathrm{~cm}^{-1}$ is indicating the $\mathrm{O}-\mathrm{H}$ stretching vibrations of hydroxyl groups. The band at $1598.58 \mathrm{~cm}^{-1}$ shows the presence of C-C stretching in the graphitic domain in the sample. The absorption at $2916.88 \mathrm{~cm}^{-1}$ originates from the aliphatic groups in coal samples [8]. Absorptions at 998.26 $\mathrm{cm}^{-1}$ is attributed to the $\mathrm{C}-\mathrm{O}$ stretching vibrations and those at $1252.34 \mathrm{~cm}^{-1}$ are due to C-O-C stretching. The peak at 1439.95 $\mathrm{cm}^{-1}$ is due to the $\mathrm{C}-\mathrm{OH}$ bond stretching [8]. The spikes between 998.26 and $600.42 \mathrm{~cm}^{-1}$ are attributed to the C-H loop bending vibration and they also indicate aromatic structure in the coal $[8,22]$.

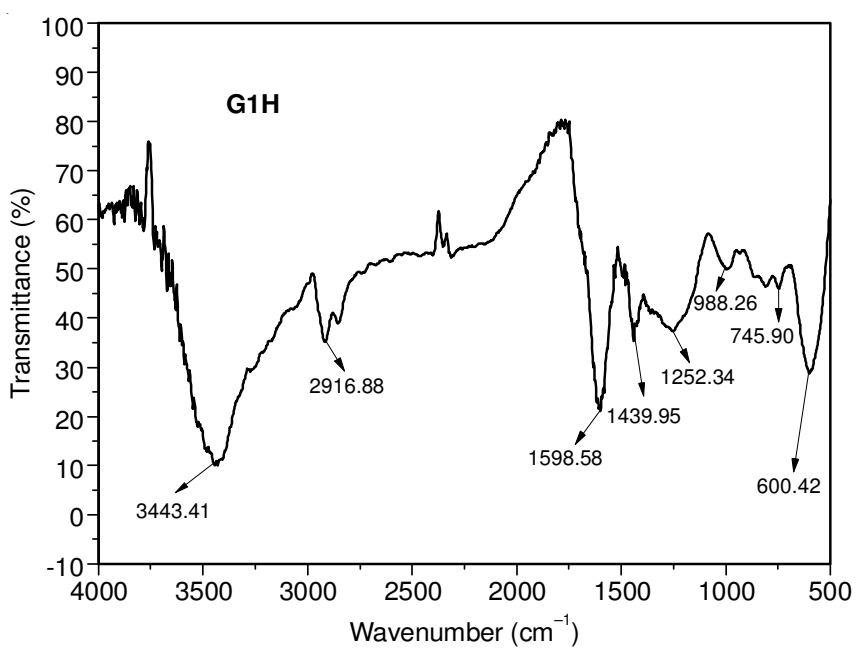

Fig. 4. FTIR analysis of graphene structure from coal

The SEM image of the obtained graphene layers is shown in Fig. 5. The surface reveals the formation of layer like structure with average size of about $43.13 \mathrm{~nm}$. There is a formation of large number of carbon layers with different shape in the nanometer scale range. This confirms the formation of layered nanocarbon of less than eight layers in the coal after acidic oxidation. The elemental analysis by EDS (Fig. 5) confirms the presence of carbon and oxygen in the layer structure indicating the formation of graphene oxide layers $(\mathrm{C}=83.13 \mathrm{wt} \%, \mathrm{O}=$ 16.87 wt \%).

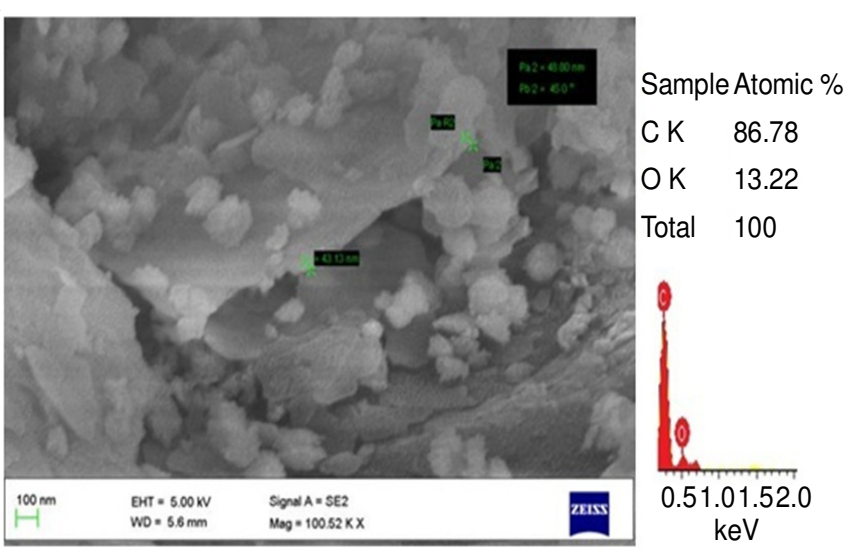

Fig. 5. Surface morphology of grapheme structure extracted from coal and EDS analysis

Transmission electron microscopic analysis: The TEM image of the nanocarbon obtained from the sub-bituminous coal is presented in Fig. 6. During the detailed electron microscopic analysis (HRTEM), presence of carbon nanomaterial in the leached product of coal are noticed. Spherical carbon dots having diameter in the range about 15 to $5 \mathrm{~nm}$ is formed in the sample.

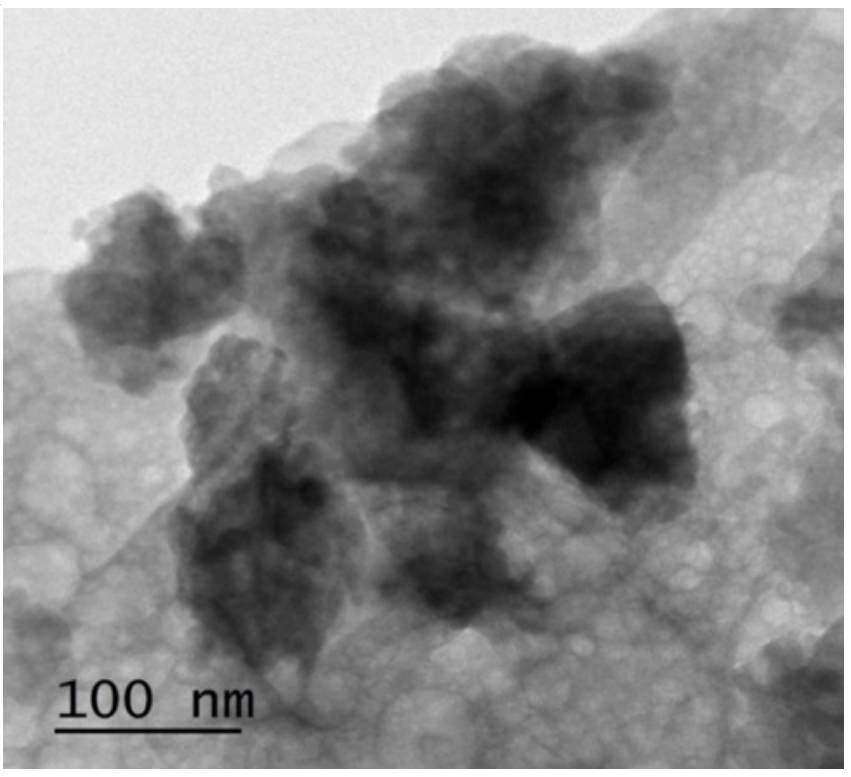

Fig. 6. TEM micrograph of sub-bituminous coal $(\mathrm{G} 1 \mathrm{H})$

Formation of graphene dots in the shape of hexagonal, spherical, graphene layers and corn shaped carbon nano tubes are noticed. Carbon nanotube in form Y-junction and bamboo are also observed in the micrograph. At some part, clustering spheres and formation of multilayer and honey comb structure are noticed. This is owing to the van der Waals forces leading to the agglomeration of carbon dots [3,6]. The TEM analysis confirms the formation of mixed phase structure of carbon nanomaterial in the leached product of sub-bituminous coal. It is concluded that bituminous coal is an effective precursor for carbon dots of size less than $10 \mathrm{~nm}$. From the sub-bituminous coal could easily extract graphene layers and carbon nanotubes whereas the lignite is a potential precursor for multilayer graphene. 


\section{Conclusion}

In conclusion, we report a facile synthesis of mixed phase graphene nanostructures from low grade coal by the method of acidic leaching. XRD results confirm the formation of mixed phase graphene nanostructures with 5 layers and hence prove turbostratic structure of coal. D2 peak at $1387 \mathrm{~cm}^{-1}$ in Raman spectra prove the presence of $s p^{2}$ and $s p^{3}$ bond-amorphous carbon structures. The broad $2 \mathrm{D}$ region also support that obtained sample is a mixed phase graphene nanostructure with multi layers. Defect to graphitic ratio and $\left(\mathrm{I}_{\mathrm{D}} / \mathrm{I}_{\mathrm{D}}\right)$ ratio was found to be 0.54 and 3.40 , respectively confirming the presence boundary like defects in the mixed phase graphene nano system. The crystalline carbon within the coal structure is displaced during oxidation, resulting in the formation of mixed structure nanometer-sized graphene with amorphous carbon. The average plane dimension parameters of the random layered graphene sheets shows that $\mathrm{L}_{\mathrm{a}}$ and $\mathrm{L}_{\mathrm{c}}$ of sub-bituminous coal were 4.82 and $1.41 \mathrm{~nm}$, respectively. The SEM morphology of the sample revealed the formation of graphitic layers in the sample. The TEM analysis confirms the formation of mixed phase structure of carbon nanomaterial in the leached product of sub-bituminous coal. The synthesis of mixed graphene structure with controllable defect could lead to new advances in the utility of coal as a novel material.

\section{REFERENCES}

1. B. Manoj, Russian J. Phys. Chem. A, 89, 2438 (2015); https://doi.org/10.1134/S0036024415130257.

2. A.K. Geim, Science, 324, 1530 (2009); https://doi.org/10.1126/science. 1158877 .

3. A.V. Ramya, B. Manoj and A.N. Mohan, Asian J. Chem., 28, 1031 (2016); https://doi.org/10.14233/ajchem.2016.19577.

4. A.V. Ramya, A.N. Mohan and B. Manoj, Mater. Sci. Poland, 34, 330 (2016);

https://doi.org/10.1515/msp-2016-0061.
5. K. Krishnamoorthy, M. Veerapandian, K. Yun and S. Kim, Carbon, 53, 38 (2013); https://doi.org/10.1016/i.carbon.2012.10.013.

6. R. Ye, C. Xiang, J. Lin, Z. Peng, K. Huang, Z. Yan, N.P. Cook, E.L. Samuel, C. Hwang, G. Ruan, G. Ceriotti, A.O. Raji, J.M. Tour and A. Marti, Nat. Commun., 4, 2943 (2013); https://doi.org/10.1038/ncomms3943.

7. Y. Dong, J. Lin, Y. Chen, F. Fu, Y. Chi and G. Chen, Nanoscale, 6, 7410 (2014); https://doi.org/10.1039/C4NR01482K.

8. B. Manoj, Int. J. Miner. Metall. Mater., 21, 940 (2014); https://doi.org/10.1007/s12613-014-0993-7.

9. C.D. Elcey and B. Manoj, Asian J. Chem., 28, 1557 (2016); https://doi.org/10.14233/ajchem.2016.19750.

10. Q. Zhou, Z. Zhao, Y. Zhang, B. Meng, A. Zhou and J. Qiu, J. Energy Fuels, 26, 5186 (2012); https://doi.org/10.1021/ef300919d.

11. B. Manoj and A.G. Kunjomana, Russ. J. Appl. Chem., 87, 1726 (2014);

12. B. Manoj and A.G. Kunjomana, J. Min. Mater. Charact. Eng., 9, 919 (2010); https://doi.org/10.4236/immce.2010.910067.

13. A.C. Ferrari and J. Robertson, Phys. Rev. B, 63, 121405 (2001); https://doi.org/10.1103/PhysRevB.63.121405.

14. W.S. Bacsa, J.S. Lannin, D.L. Pappas and J.J. Cuomo, Phys. Rev. B, 47, 10931(R) (1993); https://doi.org/10.1103/PhysRevB.47.10931.

15. K. Ramya, J. John and B. Manoj, Int. J. Electrochem. Sci., 8, 9421 (2013).

16. A. Eckmann, A. Felten, A. Mishchenko, L. Britnell, R. Krupke, K.S. Novoselov and C. Casiraghi, Nano Lett., 12, 3925 (2012); https://doi.org/10.1021/n1300901a.

17. M.A. Pimenta, G. Dresselhaus, M.S. Dresselhaus, L.G. Cançado, A. Jorio and R. Saito, Phys. Chem. Chem. Phys., 9, 1276 (2007); https://doi.org/10.1039/B613962K.

18. J. Schwan, S. Ulrich, V. Batori, H. Ehrhardt, and S.R.P. Silva, J. Appl. Phys., 80, 440 (1996); https://doi.org/10.1063/1.362745.

19. B. Manoj, Asian J. Chem., 26, 4553 (2014); https://doi.org/10.14233/ajchem.2014.15150.

20. B. Manoj, Int. J. Coal Sci. Technol., 3, 123 (2016); https://doi.org/10.1007/s40789-016-0134-1.

21. A.C. Ferrari and D. Basko, Nat. Nanotechnol., 8, 235 (2013); https://doi.org/10.1038/nnano.2013.46.

22. B. Manoj and C.D. Elcey, J. Univ. Chem. Technol. Metall., 45, 385 (2010). 